\title{
Las empresas vascas en China y la política de internacionalización del gobierno vasco
}

\author{
Eguzki Urteaga \\ Universidad del País Vasco \\ eguzki.urteaga@ehu.es
}

Resumen: este artículo analiza la presencia de las empresas vascas en China y la politica de internacionalización elaborada y posteriormente implementada por el Gobierno Vasco para propiciar dicha presencia a través de políticas públicas ambiciosas y novedosas. La necesidad para las empresas vascas de implantarse en China resulta de la globalización de la economía y de la apertura creciente de los mercados, del enorme mercado potencial que representa ese país-continente de 1.300 millones de habitantes, con el acceso de 300 millones de chinos a la clase media, y del estancamiento de la economía europea como consecuencia de la crisis económica que se inicia en septiembre de 2008. Se trata de un mercado que ofrece numerosas oportunidades; las empresas vascas disponen de recursos para poder implantarse y desarrollarse en él, si bien se enfrentan a una serie de obstáculos de indole político-administrativa, socioeconómica, cultural y lingüistica que conviene no infravalorar.

Palabras clave: empresa, País Vasco, China, Gobierno Vasco, política pública, internacionalización.

Abstract: This article analyzes the presence of Basque companies in China and the internationalization policies elaborated and later implemented by the Basque Government to propitiate the above mentioned presence thanks to ambitious and new public policies. The need for the Basque companies to be implanted in China ensues from the economical globalization and the increasing opening of markets, the enormous potential market that represents this country-continent of 1.3 billions of inbabitants with the access of 300 million Chinese to the middle class, and the stagnation of the 
European economy as consequence of the economic crisis that begins in September, 2008. But, if this market offers numerous opportunities and the Basque companies have resources to be implanted and developed in it, they have to face to politicaladministrative, socioeconomic, cultural and linguistic obstacles, that it is necessary to not underestimate.

Key words: Company; Basque Country; China; Basque Government; public policies; internationalization. 


\section{Introducción}

Este artículo analiza la presencia de las empresas vascas en China y la política de internacionalización elaborada y posteriormente implementada por el Gobierno Vasco para propiciar dicha presencia a través de políticas públicas ambiciosas, novedosas y potencialmente eficaces. De hecho, la presencia vasca en ese paíscontinente aumenta notablemente a partir de 2005, antes de verse afectada por la crisis financiera y posteriormente económica de 2008. En ese sentido, la decisión de las empresas vascas de implantarse y desarrollarse en el principal país asiático no resulta de la crisis, sino que es anterior a ella (SPRI, 2009). De la misma forma, la política de internacionalización del Gobierno Vasco se inicia a partir de la década de 1980 con los esfuerzos para promover la imagen exterior de la Comunidad Autónoma Vasca (CAV), reforzar la cooperación interempresarial para atraer inversores extranjeros a Euskadi y propiciar la apertura de las empresas vascas hacia el exterior (Gobierno Vasco, 2014a: 11).

En ese sentido, el principal motivo para implantarse en China es el mercado potencial que representa este país de 1.370 millones de habitantes, de los cuales 300 millones han accedido a la clase media y 500 millones han salido de la pobreza. Con un crecimiento económico anual que ha superado el $10 \%$ durante muchos años y una tasa de crecimiento del 7,7 \% en 2013 (OID, 2014: 5), China se ha convertido en la segunda potencia económica mundial, detrás de los Estados Unidos, además de ser el primer exportador y poseer las reservas de cambio más elevadas del planeta (Santander Trade, 2015). En un intento por adecuar su modelo socioeconómico para que sea más sostenible social y económicamente, el Gobierno chino desea fomentar el consumo interno y lo está consiguiendo poco a poco. De hecho, en 2013, el 54,4\% del crecimiento del PIB en la primera mitad del año estaba impulsado por el consumo privado y público (OID, 2014:5). Esto significa que el mercado chino, ya de por sí importante, se irá incrementando conforme pase el tiempo (Borgonjon, 2009), lo que convierte la implantación en China en un reto estratégico para numerosas empresas europeas, y, entre ellas, las vascas (Ceiss Consultores, 2009).

No en vano, es evidente que la crisis económica que azota el continente europeo está obligando las empresas vascas a buscar nuevos mercados, especialmente aquellos que no se ven afectados sobremanera por la crisis económica. Y, como anticipa el propio Gobierno Vasco, «los próximos años vendrán caracterizados por una tónica similar, con crecimientos en geografías lejanas y un proceso de recuperación débil en nuestro entorno más inmediato» (Gobierno Vasco, 2014a: 17). En ese sentido, la CAV no es ajena a la crisis económica que afecta al continente europeo en general y a los países del sur del Viejo Continente en particular. 
Su dependencia hacia el mercado español y europeo conlleva que se vea afectada por el retroceso del consumo interno, de los intercambios comerciales con el resto del Estado y de las exportaciones hacia Europa. Esta situación resulta a la vez de la crisis financiera y de la gestión de dicha crisis, que ha sido sinónimo, en la mayoría de los países europeos y de manera simultánea, de la aplicación de políticas de austeridad que, lejos de propiciar la recuperación económica, han sumergido los países concernidos en la recesión o, al menos, en una fase de escaso crecimiento.

Ante esta situación, el Gobierno Vasco ha decidido reforzar su política de internacionalización de las empresas vascas propiciando la mejora de su competitividad (Larrea, 2008) y la búsqueda de nuevos mercados. Esa política obedece a la Estrategia de las «4I» para la Reactivación Económica, que pretende acompañar dichas empresas en su proceso de adaptación a un entorno competitivo global en evolución permanente (Gobierno Vasco, 2014a: 2). Precisamente, la política de promoción económica del Gobierno Vasco se articula en torno a cuatro factores competitivos clave: la innovación, «como fuente de generación de conocimiento y de transformación del mismo en valor económico y social»; la industrialización, «basada en la recuperación del espíritu industrial y el decidido apoyo a la economía productiva»; la inversión, «como palanca de competitividad para el impulso de la $\mathrm{I}+\mathrm{D}+\mathrm{i}$, la generación y transferencia de tecnología y conocimiento, y el desarrollo de infraestructuras y proyectos estratégicos»; y la internacionalización, «como la mejor garantía para diversificar los riesgos y mejorar la competitividad global de las empresas vascas» (Gobierno Vasco, 2014a: 2).

Esta ayuda del Gobierno Vasco para facilitar la internacionalización de las empresas vascas es aún más necesaria puesto que estas se enfrentan a una serie de obstáculos a la hora de implantarse en China y desarrollar su actividad económica en ese país.

\section{Características del mercado chino y coyuntura económica}

Ante todo, es preciso detenerse sobre las características del mercado chino. Cabe señalar que la economía diversificada del gigante asiático está dominada por los sectores de la industria manufacturera y la construcción, que aportan casi la mitad del PIB. «China se ha convertido en uno de los destinos favoritos para el traslado de unidades manufactureras mundiales a causa del bajo coste de la mano de obra, aunque su valor está en aumento» (Santander Trade, 2015). El crecimiento económico ha coincidido principalmente con el desarrollo de un sector 
manufacturero competitivo y orientado a la exportación. Más de la mitad de las exportaciones chinas son realizadas por empresas con capital extranjero y su participación en el valor agregado sectorial varía según el sector: desde más del $60 \%$ para la electrónica hasta menos del $20 \%$ para la mayoría de los bienes intermedios. «Es reseñable que el sector estatal todavía contribuye aproximadamente al $40 \%$ del PIB» (Santander Trade, 2015).

Por otro lado, aunque obstruido por los monopolios públicos y las regulaciones restrictivas, el sector terciario crece notablemente dado que su cuota en el PIB se eleva a más del $45 \%$ y emplea a un tercio de la población activa (Santander Trade, 2015).

De la misma forma, a pesar de que solo aporte alrededor del $10 \%$ del PIB, la agricultura emplea a cerca del $35 \%$ de la población activa. «China es uno de los mayores productores y consumidores de productos agrícolas. El país ocupa el primer lugar en la producción mundial de cereales, arroz, algodón, patatas y té» (Santander Trade, 2015). En cuanto a la ganadería, China domina también la producción ovina, porcina y de especies marinas. Una serie de planes tienen como objetivo transformar la agricultura, modernizándola, diversificándola y volviéndola más productiva (Santander Trade, 2015).

El sector de la minería desempeña igualmente un papel importante en la economía china, ya que el país cuenta con un subsuelo rico en recursos energéticos. «China tiene grandes reservas de carbón (la primera fuente de energía del país), que representa dos tercios del consumo total de energía primaria» (Santander Trade, 2015). El país es líder mundial en la producción de ciertos minerales (estaño, hierro, oro, fosfatos, zinc y titanio) y tiene importantes reservas de petróleo y gas.

De manera más coyuntural, es necesario recordar que la recesión mundial de 2008 ha ralentizado el ritmo de crecimiento que había experimentado el país y ha puesto de manifiesto los límites de un crecimiento basado esencialmente en las exportaciones (Santander Trade, 2015). Como consecuencia de la desaceleración económica mundial y la bajada de los intercambios comerciales, el crecimiento chino se ha desacelerado en 2015, siendo inferior al $7 \%$ debido a la morosidad del mercado inmobiliario y del sector de la construcción y a la dependencia hacia la demanda externa, los gastos de inversión y la expansión del crédito.

Precisamente, esa dependencia hacia el exterior, el fuerte endeudamiento público, la opacidad del sistema financiero y las menores perspectivas de crecimiento económico han provocado cierto nerviosismo entre los inversores nacionales e internacionales, lo que ha conllevado una contracción de las bolsas chinas. La decisión del Gobierno chino de devaluar el yuan en agosto de 2015 ha encendido 
todas las alarmas ya que ha sido interpretada por los mercados financieros como un síntoma de debilidad superior a lo que apuntaban los indicadores oficiales. En ese sentido, «la falta de credibilidad de las autoridades chinas y su manera opaca de manejar la economía están influyendo mucho en la reacción de los inversores» (Cuesta, 2015). Más allá de estos sobresaltos bursátiles, esta desaceleración económica ha sido impulsada por el propio Gobierno chino, que desea proceder a un cambio estructural de su modelo de crecimiento para orientarse hacia una modelo más sostenible ${ }^{1}$.

\section{Las empresas vascas en China}

En ese contexto, hoy en día 187 empresas vascas están implantadas en China, entre las que figuran el grupo Corporación Mondragón (MCC), Orbea, Forjas Iraeta, Gestamp, Orkli, Gamesa u Ormazabal (Efe, 2012). Según un estudio realizado por la Sociedad para la Promoción y Reconversión Industrial (SPRI) en 2009, la mayoría de las empresas vascas se concentran, en ese orden, en las regiones de Shanghái, Jiangsu y Beijing (Pekín). Las empresas productivas se ubican mayoritariamente en la región de Jiangsu, mientras que las sociedades comerciales prefieren implantarse en Shanghái y, en menor medida, en Beijing. Por último, las empresas de servicios privilegian las dos principales metrópolis chinas. En ese sentido, la práctica totalidad de las empresas vascas están ubicadas en la costa oeste de China, que es la zona más dinámica y próspera del país.

En cuanto al período y ritmo de implantación de las empresas vascas en China, resulta que las instalaciones son limitadas y constantes entre 1990 y 2004 (Basterra, 2008), antes de aumentar repentinamente a partir de 2005 para decrecer de nuevo en 2008. En otros términos, los años 2005, 2006 y 2007 fueron especialmente propicios a la instalación de dichas empresas en el principal país asiático. Estas implantaciones se produjeron en un contexto de fuerte crecimien-

1 Conviene subrayar que, durante el tercer plenario de los miembros del Comité Central del Partido Comunista de China (PCC) a finales de 2013, «el PCC anunció su programa de reforma de aquí a 2020» (Santander Trade, 2015). Este incluye: avances políticos, económicos y sociales como el cierre de los centros de trabajos forzados; una mayor independencia de los tribunales a nivel local; la flexibilización de la política del hijo único; la reforma del bukou, que regula la movilidad y el acceso a los servicios públicos por los ciudadanos; la reforma del acceso a bienes raíces para facilitar la cesión de tierras agrícolas por los campesinos; la liberalización de las tasas de interés; la apertura progresiva de las transacciones financieras; y la reforma de las empresas del Estado (Santander Trade, 2015). El interés exclusivo por el crecimiento económico ha dado lugar a una preocupación creciente por problemas sociales como la degradación del medio ambiente, la corrupción o el aumento de las desigualdades sociales (Santander Trade, 2015). De hecho, todavía persisten numerosos desafíos relacionados con el envejecimiento de la población y la existencia de grandes diferencias entre los niveles de vida de la ciudad y el campo, entre las zonas urbanas costeras y el interior y el oeste del país, y entre las clases medias urbanas y los olvidados por el crecimiento (Santander Trade, 2015). Estas desigualdades preocupan cada vez más a las autoridades chinas y a los inversores. Si bien la pobreza ha disminuido considerablemente en China, sigue afectando a casi el $10 \%$ de la población, que vive con menos de un dólar al día. 
to, tanto en la CAV como en China, y de deseo de las compañías vascas de conquistar nuevos mercados. En ese sentido, China aparece como un mercado donde es fundamental implantarse y desarrollarse dado el fuerte potencial y las expectativas de crecimiento que ofrece ese país (Lemoine y Hernandez, 2007). En 2008, sin embargo, esa tendencia se atenúa de nuevo como consecuencia de la crisis, aunque las empresas vascas continúen apostando por ampliar su presencia en el mercado chino (Diez Mon, 2014). De hecho, según el Gobierno Vasco, «la actividad exportadora puede haber contribuido a la supervivencia de muchas empresas [vascas], ya que las empresas con actividad internacional han experimentado un menor retroceso que el tejido empresarial general» (Gobierno Vasco, 2014aः 38). En ese sentido, la estrategia de internacionalización constituye un vector de refuerzo competitivo y de defensa del empleo.

Más recientemente, se observa una tendencia de ciertas empresas vascas a repatriar parte o totalidad de su producción de China. Es el caso de NBI Bearings. Esta empresa nacida hace trece años en Vizcaya, que se trasladó posteriormente a Álava, es «fruto de la iniciativa de dos profesionales con experiencia en la comercialización de rodamientos y con el objetivo de producir este tipo de productos pero en pequeñas series y para prestaciones exigentes» (Álvarez, 2015). Decidió inicialmente mantener su sede y su departamento de Investigación y Desarrollo en el País Vasco y trasladar su planta productiva a China, donde los costes de producción son inferiores. Así, «con el esquema dual de la investigación en Okondo y una pequeña planta de producción propia en China, NBI ha desarrollado durante estos años un modelo de éxito» (Álvarez, 2015). No en vano, ha decidido repatriar su producción como consecuencia del incremento de los salarios en China, la competencia creciente de las empresas asiáticas, los aranceles que los Estados Unidos aplican a los productos chinos y la devaluación del euro con respecto al dólar.

En cualquier caso, entre las principales razones mencionadas por las empresas vascas para implantarse en China figuran las siguientes (SPRI, 2009):

- Incrementar la capacidad de la cadena de suministro.

+ Estar cerca de los clientes habituales.

+ Realizar ahorros de escala.

- Completar la gama de productos.

- Beneficiarse de la flexibilidad productiva para adaptarse a los imperativos del mercado.

+ Beneficiarse de la logística y de las infraestructuras. 
- Teniendo en cuenta las razones principales por las cuales las empresas vascas se implantan en China, junto con el destino de sus ventas y los competidores, podemos distinguir varios tipos de empresas en función de las estrategias (Luzarraga, 2006) diseñadas para implantarse y desarrollarse en el gigante asiático (SPRI, 2009):

- Las empresas que producen en China para vender principalmente en el mercado chino y asiático, bien a clientes chinos, bien a multinacionales que están instaladas en esta región del mundo.

- Las empresas que producen en China para abaratar los costes y suministrar principalmente a sus mercados en Euskadi, España y Europa. Se apoyan en los menores costes de producción y, sobre todo, en los costes laborales inferiores para ganar en competitividad-precio.

- Las empresas que tienen una oficina en China orientada a vender o comprar en dicho mercado. En ese caso, se desarrolla una actividad comercial y de marketing dirigida a identificar, negociar y comprar a proveedores chinos o a dar a conocer productos, servicios y procesos vascos a clientes chinos (SPRI, 2009).

- Las empresas de servicios que prestan sus servicios a empresas europeas que operan en China o directamente a empresas chinas implantadas en el país y que desean ampliar su actividad. Es cuestión de asesorar y formar a profesionales para que puedan incrementar su facturación.

En general, como subraya la SPRI, «las empresas que van a vender a China son más grandes y tienen instalaciones en más países, mientras que las que van a China por motivos de coste (para comprar o producir barato) son más pequeñas y, en la mayoría de los casos, China es su única implantación» (SPRI, 2009). Según el Gobierno Vasco, el universo de las empresas exportadoras vascas es reducido, con una importante concentración del valor de la exportación en empresas medianas $y$ grandes.

Vinculado a todo ello, se ha producido un cambio progresivo y rápido a la vez en los productos fabricados por las plantas chinas de las empresas vascas. En efecto, durante un largo período se asoció China con la fabricación y posterior exportación de productos de nulo o escaso valor añadido que exigían una amplia mano de obra. Nada más lejos de la realidad, dado que, si el $21 \%$ de las empresas vascas solo monta o produce componentes intensivos en mano de obra y el $16 \%$ fabrica productos completos pero de tecnología menos avanzada, el resto, es decir, el 63\%, recurre a tecnología avanzada (SPRI, 2009). Así, el $40 \%$ fabrica productos complejos y tecnológicamente avanzados, con tecnología desarrollada 
por la matriz vasca; el $11 \%$ produce productos completos y tecnológicamente avanzados, con tecnología desarrollada en China; y el $11 \%$ restante fabrica productos completos y tecnológicamente avanzados, desarrollada conjuntamente entre China y la matriz vasca.

Ese cambio traduce la apuesta decidida del Gobierno chino y de las empresas chinas por atraer a empresas tecnológicamente avanzadas, gracias a políticas de aranceles adecuadas, y por privilegiar la transferencia tecnológica asociada a la firma de contratos. El objetivo de China es reducir lo antes posible la distancia tecnológica que separa las empresas europeas y las chinas. Asociado a ello, el Estado chino ha apostado decididamente por la inversión en educación y en $\mathrm{I}+\mathrm{D}+\mathrm{i}$ con vistas a elevar el nivel de cualificación y las competencias profesionales de la población y aumentar notablemente el número de ingenieros y doctores. De hecho, actualmente China forma a más doctores que los Estados Unidos y Europa.

Ante estas transformaciones, algunas empresas vascas prefieren repatriar su producción. Es el caso de las cooperativas Orbea y Fagor Industrial de la Corporación Mondragón (Altuna y Urteaga, 2014a), que han decidido cerrar sus fábricas en China para concentrar las inversiones en otras plantas más productivas. Fagor Industrial, cooperativa dedicada a la fabricación de cocinas industriales, equipación de lavandería y frío, ha adoptado la decisión de «racionalizar sus centros productivos» (EITB, 2015) y ha apostado por potenciar las plantas de las que dispone en México, Turquía y Euskadi. La planta ubicada en el parque empresarial de Kunshan, una población próxima a Shanghái, se cerró después de que la firma llegara a la conclusión de que no cumplía los «rangos de competitividad suficientes en esos mercados» (EITB, 2015). En ese mismo parque empresarial que impulsó y financió MCC en 2007, estaba instalada una planta del fabricante de bicicletas Orbea, que también ha decidido clausurarla a partir del 30 de junio de 2015 como consecuencia de la redefinición de sus estrategias de fabricación. Esta firma ha decidido potenciar la producción en otras plantas como la de Mallabia (Vizcaya), donde «se verá incrementada la capacidad de customización tanto en pinturas como en opciones de componentes» (EITB, 2015).

Pero la mayoría de las empresas vascas se mantienen en China, donde incrementan sus ventas. Es el caso del sector de la automoción, que apuesta decididamente por producir en el país asiático ${ }^{2}$, de manera que cooperativas como Fagor

2 A ese respecto, es preciso recordar que la industria de la automoción es un sector esencial de la economía vasca. Su gran desarrollo permite encontrar prácticamente 300 empresas relacionadas con ese sector, que fabrican un tercio del total de los componentes del Estado español, y dos grandes fabricantes: Mercedes Benz, en Vitoria-Gasteiz, y el Grupo Irizar, con sede en Ormaiztegi. Esta gran concentración en un pequeño territorio como el del País Vasco solo es comparable a la de algunos Länder alemanes. El sector se ha convertido en estratégico para la economía vasca, junto con la energía y la aeronáutica (Confebask, 2014). Ha contribuido, unido al acero, a mantener el importante peso de la industria en el PIB de la Comunidad Autónoma Vasca (el 24 \% en 2013). En 2013, el segmento del automóvil facturó en el País Vasco 13.071 millones de euros, un 
Ederlan (Altuna y Urteaga, 2014b), Batf y Cikautxo mantienen sus fábricas en Kunshan. En 2014, las cooperativas del grupo Mondragón implantadas en China facturaron en aquel país un total de 350 millones de euros, mientras que la Corporación mantiene su expectativa de duplicar estas cifras para el año 2020 (EITB, 2015). Asimismo, como indica José Luis Gil, director de Internacionalización de la Cámara de Comercio de Bilbao, «la mayor parte de las operaciones vascas en China son inversiones de empresas de relevancia que se han implantado en el país para suministrar al mercado interno. Lo que, unido a que gran parte del comercio vasco está ligado a los componentes de maquinaria que será necesaria a medio plazo para impulsar el cambio de [modelo] económico, alimenta las expectativas [positivas] de las compañías» (Diez Mon, 2015).

Entre las empresas vascas que mayor éxito han tenido en China figura $\mathrm{Ga}$ mesa, cuya sede se encuentra en Zamudio (Vizcaya). El fabricante de turbinas inició sus actividades en China en 2005 con la instalación de una fábrica en Tianjin (cerca de Beijing). Su crecimiento ha sido notable desde entonces, contando en la actualidad con media docena de centros productivos en el país. «Gamesa supo comprender la potencialidad de China en su sector y ha tenido la capacidad para llevar a cabo un fuerte crecimiento en un período de tiempo muy corto» (Iberchina, 2016). Cuatro de sus centros productivos están en Tianjin, que se ha convertido en la mayor base productora de la empresa fuera de España. Los planes de inversión de Gamesa en China suponen una inversión acumulada de 130 millones de euros en 2012 (Iberchina, 2016). Gamesa ha sabido posicionarse en uno de los países del mundo con mayor crecimiento de la energía eólica ya que, en 2011, China supuso el 30\% de los megavatios vendidos en el mundo. «Gamesa actúa en una doble vertiente: como suministrador de turbinas, que se encuentran en más de 60 parques de China, y como operador de parques eólicos» (Iberchina, 2016). Para esta última línea, la empresa se ha aliado con empresas locales. En 2010, por ejemplo, se puso en marcha el parque de Taipingshan, desarrollado en asociación con la empresa china China Guandong Nuclear Wind Corporation.

5,2\% más que en 2012, cuando alcanzó los 12.400 millones, y 2.000 más que antes de la crisis. Los datos de los últimos años sitúan el peso de la industria vasca de la automoción en un 17\% del PIB del País Vasco; el $20 \%$ si se suman los datos de Mercedes Benz en Vitoria-Gasteiz (Confebask, 2014). Además, el $90 \%$ de la facturación automovilística del País Vasco se exporta, lo que contribuye al superávit comercial vasco; más de la mitad se produce fuera de Europa; y da empleo en los Territorios Históricos Vascos de Vizcaya, Guipúzcoa y Álava a prácticamente 35.000 personas. 


\section{Oportunidades y obstáculos del mercado chino}

Como hemos mencionado anteriormente, el mercado chino ofrece numerosas oportunidades (Peters, 2010) relacionadas con la importancia de su mercado interior $y$ las perspectivas de crecimiento que ofrece. Efectivamente, con una población de 1.370 millones de habitantes (con una tasa de crecimiento anual del $0,5 \%$ ) y con una renta per cápita en fuerte aumento (9.040 dólares) gracias a un crecimiento económico notable y sostenido, el mercado potencial para las empresas vascas es enorme. Si a ello le añadimos la fortaleza del sector industrial y el rápido desarrollo del sector servicios, China ofrece numerosas oportunidades.

A su vez, los cambios políticos han sido notables desde el acceso al poder de Deng Xiaoping y el lanzamiento de la «política de reforma económica y apertura al exterior», que supuso el inicio del proceso gradual de introducción de elementos de la economía de mercado en China. En 1987, se acuña la doctrina de la «fase inicial del socialismo», que confiere una base teórica al proceso de reforma económica según el cual «el único rasero para medir el trabajo del Partido Comunista Chino es su contribución al desarrollo de las fuerzas productivas» (OID, 2014: 2). Abre la puerta a la legalización de la propiedad privada de los medios de producción, que se consagra un año más tarde con la reforma de la Constitución. Esta autoriza la creación de un sector económico privado como «complemento» del sector público. Las reformas emprendidas han propiciado el proceso de crecimiento económico sostenido que ha posibilitado la creación de una clase media y el desarrollo del mercado interior.

Entre las restantes oportunidades generadas por el mercado chino figuran la planificación de grandes transformaciones en los ámbitos agroalimentario, energético y medioambiental; la presencia de multinacionales, que valoran las marcas europeas y los proveedores que aportan calidad e innovación; la sofisticación de la demanda a raíz del incremento del nivel de exigencia de los consumidores chinos, lo que aumenta las oportunidades de las empresas vascas; la ubicación estratégica de China, que la convierte en una plataforma apropiada para vender en el resto de Asia; el dinamismo empresarial y la fuerte capacidad inversora de las empresas chinas que gozan de liquidez; la gran variedad de proveedores que ofrecen precios competitivos; $y$ los costes competitivos en materia de mano de obra, materiales, maquinaria y suelo, aunque se hayan incrementado notablemente durante los últimos años (SPRI, 2009).

Pero conviene no olvidar otros factores tales como la abundancia de mano de obra cualificada como consecuencia de la política educativa elaborada e implementada por las sucesivas generaciones de dirigentes chinos; las buenas comunicaciones e infraestructuras asociadas a la elevada competencia y disponibili- 
dad de los operadores logísticos y de transporte; la construcción de importantes infraestructuras en materia de carreteras, puertos, aeropuertos y trenes de alta velocidad; la gran capacidad de inversión gracias a una estrategia de reinversión sistemática de los beneficios realizados; la estabilidad política y la planificación de la actividad económica con actuaciones relevantes que se programan y que se ejecutan de manera rigurosa; o la visión a medio y largo plazo de los dirigentes políticos del país, lo que permite a China tener un estrategia de desarrollo coherente (SPRI, 2009).

Simultáneamente, el mercado chino es sinónimo de una serie de obstáculos de índole política, socioeconómica y cultural, empezando por la dificultad que tienen numerosos empresarios vascos para comprender la historia, el tamaño y la diversidad del país-continente que es China. En efecto, además de por su diversidad cultural y étnica, China se caracteriza por la variedad lingüística dado que, aparte del mandarín, que constituye la lengua nacional, hay otras seis lenguas reconocidas por el artículo 4 de la Constitución (OID, 2014: 1). Asimismo, en China coexisten diversas religiones. Las tres religiones tradicionales son el taoísmo (20 millones de practicantes), el budismo (100 millones de fieles) y el confucianismo, pero no hay que olvidar la presencia del islam (20 millones de creyentes) y del cristianismo en sus vertientes católica ( 5 millones de fieles) y protestante ( 5 millones de creyentes).

A este respecto, las empresas vascas se enfrentan a un conjunto de limitaciones tales como las importantes diferencias culturales a la hora de gestionar los negocios y de relacionarse con sus clientes y proveedores, empezando por la diferencia de hábitos; las peores condiciones y la menor seriedad en los pagos de las empresas chinas en comparación con sus homólogos vascos; el escaso desarrollo de la gestión basada en la calidad; el creciente proteccionismo de las autoridades chinas, que imponen restricciones y aranceles a las empresas extranjeras de escaso valor tecnológico e innovador; el retraso de las empresas del sector servicios en comparación con las empresas del sector industrial; las dificultades para contratar $y$ retener al personal cualificado, lo que provoca tensiones en ciertos sectores $y$ puestos, y la volatilidad en la gestión de la mano de obra; la escasa involucración y participación de los empleados chinos en la gestión de las empresas vascas; o las incertidumbres que pesan sobre la economía china, como pueden ser la alta inflación, el fuerte incremento de los costes de producción, la notable especulación inmobiliaria, las tensiones sociales o la elevada contaminación (SPRI, 2009).

A todo ello conviene añadir aspectos relacionados con la organización político-administrativa, sabiendo que a partir del final de la década de 1980 y, sobre todo, de la década de 1990 se produjeron una serie de cambios. Así, en 1987 se 
adoptó el principio de separación entre el Partido Comunista Chino y el Estado (OID, 2014: 2). A su vez, en 1998 se enmendó la Constitución china para incluir el concepto de «Estado socialista de derecho», lo que ha propiciado el progreso del sistema jurídico del país. Cuatro años más tarde se aprobó la «teoría de las tres representaciones», que significaba, por primera vez, la admisión de los empresarios privados en el Partido Comunista Chino, lo que lo convertía en un partido interclasista (OID, 2014:3). A pesar de estos cambios, las barreras a la actividad empresarial no han desaparecido del todo. Así, las empresas vascas que desean invertir e instalarse en China se enfrentan a la fuerte burocracia, que ralentiza los procesos y dificulta las gestiones; la existencia de complejas ramificaciones entre las administraciones estatales, regionales y locales; la mayor inseguridad jurídica y la prevalencia de cierta arbitrariedad; o el cambio repentino de leyes sin previo aviso (SPRI, 2009).

Precisamente, con vistas a aprovechar las oportunidades ofrecidas por el mercado chino y enfrentarse a los problemas con los que se pueden encontrar las empresas vascas implantadas en ese país, el Gobierno Vasco ha diseñado e implementado su política de internacionalización (Euskonews \& Media, 2015).

\section{La política de internacionalización del Gobierno Vasco}

En efecto, el Gobierno Vasco ha aprobado el Plan de Internacionalización Empresarial para el período 2014-2016 con el objetivo de que dicho Plan sea un instrumento para planificar y guiar el apoyo a la internacionalización de las empresas vascas durante la décima legislatura (Gobierno Vasco, 2014aः 2). Se trata de un plan estratégico que pretende responder a los principales retos a los que se enfrentan las empresas vascas:

- Tratándose de una economía abierta, el Gobierno Vasco ha diseñado la Estrategia Basque Country (Gobierno Vasco, 2014b), dentro de la cual el Plan de Internacionalización constituye «el instrumento de ejecución con sustantividad propia» de dicha estrategia en los ámbitos de la economía y de la industria.

- Las empresas vascas necesitan apoyo para superar una crisis que se prolonga desde hace siete años, por lo que se les ofrecen herramientas y marcos de actuación para abordar su inserción en el mercado global, sabiendo que la mayoría de las empresas vascas son pequeñas o medianas y que necesitan una ayuda adaptada a sus capacidades y necesidades (Gobierno Vasco, 2014a:3). 
- El Gobierno Vasco quiere ayudar las empresas vascas «a encontrar su espacio competitivo en la economía global [...], contemplando las diferencias de tamaño y etapa de internacionalización» (Gobierno Vasco, 2014a: 3).

- La CAV cuenta con un conjunto de actores y programas que constituyen un sistema de apoyo a la internacionalización empresarial completo y relativamente avanzado y que se enfrenta al reto de reforzar la coordinación institucional, la eficacia en la respuesta a las necesidades de las empresas y la eficiencia en el uso de sus recursos.

- El Gobierno Vasco apuesta por ejercer un «liderazgo eficaz, responsable y riguroso en la gestión» (Gobierno Vasco, 2014a: 4), abordando de manera integral las prioridades fijadas.

No en vano, el Plan de Internacionalización Empresarial 2014-2016 no es el primer esfuerzo de apoyo a la internacionalización del Gobierno Vasco. Por una parte, este plan se inscribe en la mencionada Estrategia Basque Country, a través de la Estrategia Marco de Internacionalización 2020: Euskadi Basque Country (Gobierno Vasco, 2014b), que constituye el documento de planificación estratégica en la materia para toda la legislatura. Por otra, el Departamento de Desarrollo Económico y Competitividad (DDEC) ha llevado a cabo un ejercicio de planificación de las políticas, programas e instrumentos plasmados en el Plan de Actuación 2013-2016 (Gobierno Vasco, 2014a: 5). Por último, ha desplegado medidas de impacto inmediato en relación con el nuevo diseño del programa de referencia Prointer para reforzar su eficacia, eficiencia y alcance; ha ampliado y actualizado el programa de Becas Global Training para 400 jóvenes; ha seleccionado los mercados internacionales de actuación prioritaria como China y trabaja en la concentración de la Red Exterior de SPRI (Efe, 2011) para cubrir dichos países; y ha impulsado una reflexión conjunta con las diputaciones forales y las cámaras de comercio para trabajar de manera más coordinada en el apoyo de las empresas vascas (Gobierno Vasco, 2014a: 5).

\subsection{El contexto político y normativo del Plan}

El Plan de Internacionalización Empresarial 2014-2016 se enmarca en un contexto político-normativo europeo, estatal y regional que determina las prioridades, los contenidos y las herramientas de acción definidas en este.

A nivel europeo, conviene señalar que, desde su creación, la Unión Europea ha trabajado en la eliminación de barreras comerciales entre sus países miembros. No en vano, hoy en día, dicha apertura se enfrenta a nuevos retos como el acceso 
prioritario a mercados emergentes (Gobierno Vasco, 2014a: 8). Desde el punto de vista de los mercados, la atención se centra en la apertura de nuevos mercados y el fortalecimiento de los flujos comerciales, de modo que el esfuerzo se focalice en terceros países. Entre las políticas e iniciativas más relevantes en materia de internacionalización llevadas a cabo por la Comisión Europea en los últimos años, podemos citar las siguientes (Gobierno Vasco, 2014a: 8-10):

- La Estrategia Europa 2020, cuyo fin es estimular un crecimiento inteligente, sostenible e integrador, estableciendo el liderazgo industrial como una de las líneas de actuación prioritarias para las regiones y los Estados miembros. Esta iniciativa contempla promover: 1) la internacionalización de las pymes; 2) la reestructuración de los sectores en crisis hacia actividades con perspectiva de futuro; y 3 ) la mejora del entorno empresarial.

- El Small Business Act (SBA), que pretende animar y ayudar a las pymes a beneficiarse del crecimiento de los mercados exteriores a la Unión Europea.

- El Small Business Big World, que propicia la presencia de las pymes europeas fuera del mercado único europeo acompañándolas a lo largo de todo el proceso.

- El Programa COSME, que aspira a mejorar el acceso a los mercados tanto a nivel comunitario como a escala mundial apoyando la red Enterprise Europe Network y respaldando la cooperación industrial internacional.

- La Europe Enterprise Network, que fomenta la colaboración entre empresas europeas y ayuda a las pymes a explotar al máximo las oportunidades que ofrece tanto el mercado europeo como el mercado internacional.

A nivel estatal, existe todo un arsenal jurídico que condiciona la política de internacionalización del Gobierno Vasco (Gobierno Vasco, 2014a: 10):

- La Ley de Acción y del Servicio Exterior del Estado (LASEE), cuyo objetivo principal es coordinar y alinear el trabajo de las instituciones españolas en el extranjero. El artículo 19 precisa en qué consiste «la Acción Exterior en materia económica, comercial, financiera y de apoyo a la internacionalización de la empresa».

- La Ley de Apoyo a los Emprendedores y su Internacionalización, que aspira a «apoyar el emprendedor y la actividad empresarial, [y a] favorecer su desarrollo, crecimiento e internacionalización» (Gobierno Vasco, 2014a: 10).

- La Ley Básica de las Cámaras Oficiales de Comercio, Industria, Servicios y Navegación, que ofrece nuevas oportunidades a las pymes en los 
mercados internacionales concediendo un rol especial a las cámaras que desarrollarán el Plan Cameral de Internacionalización.

En cuanto al nivel autonómico, el Gobierno Vasco ha impulsado una política de internacionalización de las empresas vascas, dado que esta constituye uno de los objetivos estratégicos de la política industrial vasca. Los primeros pasos se dieron en la década de 1980 con la intención de promover la imagen exterior de Euskadi y reforzar la cooperación interempresarial para atraer inversores extranjeros (Gobierno Vasco, 2014a: 11). Estos esfuerzos fueron liderados por la SPRI y el Departamento de Comercio, Consumo y Turismo, que llevaron a cabo proyectos puntuales y bajo demanda según la realidad y las necesidades de diversificación económica y de empleo de la época (Velasco, 1991).

En la década posterior, marcada por el proceso de integración europea, comenzó la sistematización de la actividad internacional con los planes estratégicos de promoción exterior de las empresas vascas en 1994-1997 y 1997-1999. Durante ese período, las políticas de internacionalización tuvieron un carácter multidireccional al combinar acciones de apoyo a la internacionalización de las empresas vascas y de atracción de inversores extranjeros, y al extender la acción pública de formación y sensibilización (Gobierno Vasco, 2014a: 11). Además, en esos años se inicia la política de clústeres para consolidar la cooperación interempresarial, que ha favorecido que hoy en día Euskadi cuente con una serie de clústeres industriales destacados, claves en el proceso de internacionalización de las empresas vascas (Gobierno Vasco, 2014a: 11).

En la primera década del nuevo siglo, las políticas de internacionalización del Gobierno Vasco se integraron en sucesivos planes interdepartamentales de competitividad (Plan Interinstitucional de Promoción Económica 2000-2003 y Plan de Competitividad Empresarial e Innovación Social 2005-2009). En los primeros años se sentaron las bases de las políticas de aumento de la base exportadora y de promoción de la diversificación geográfica y sectorial, enfocada fundamentalmente a la internacionalización de las pymes vascas, aún vigentes hoy en día a través de programas como Prointer (Gobierno Vasco, 2014a: 11). En la segunda mitad de la década, con el protagonismo de la innovación y la economía del conocimiento como factores clave de competitividad, las políticas de internacionalización se extienden a nuevos sectores con menor proyección en el extranjero hasta entonces, como los servicios. Además, las políticas dirigidas a las empresas industriales se refuerzan con el apoyo a la consolidación de la actividad internacional de empresas a través del impulso de la red exterior de la SPRI (Gobierno Vasco, 2014a: 11). 
Por último, el Plan de Competitividad Empresarial 2010-2013 (PCE), antecedente del último Plan, continúa apostando por la internacionalización como palanca fundamental de competitividad, y fija como uno de sus tres ejes estratégicos de acción el posicionamiento de la CAV como una economía abierta, impulsando la apertura al exterior de las empresas vascas: "Con el impacto de la crisis económica, en los últimos años se inició en ámbitos como la captación en internacionalización, con el lanzamiento del programa Global Training, o la coordinación interinstitucional, con la creación del Consejo de Internacionalización del País Vasco» (Gobierno Vasco, 2014a: 11).

\subsection{El sistema vasco de internacionalización de empresas}

Las empresas vascas que desean internacionalizarse solicitan apoyo para llevar a cabo sus actividades de promoción, financiación, asesoría experta, acompañamiento, formación y capital humano (Gobierno Vasco, 2014a: 50). En función del grado de internacionalización de la empresa, estas demandas se acotan en necesidades más específicas, desde la búsqueda de actores en el exterior de las empresas que inician la exportación hasta la selección y contratación de personal destinado a las empresas que abordan la implantación productiva en China (Gobierno Vasco, 2014a: 50). Las empresas vascas buscan apoyo para la internacionalización principalmente en la SPRI (Arriola, 2015: 44), las cámaras de comercio, el ICEX y la Dirección de Internacionalización del Gobierno Vasco. Mientras que las empresas no implantadas en el exterior se apoyan más en las cámaras de comercio y la SPRI, las ya instaladas solicitan la ayuda de la SPRI, el ICEX y el Gobierno Vasco.

Esa labor de apoyo a la internacionalización es llevada a cabo tanto en el País Vasco como en China. Así, en septiembre de 2015, una delegación de la SPRI visitó China con un triple objetivo: 1) conocer de primera mano la situación de las empresas vascas implantadas en este país; 2) analizar cuál es su situación tras los cambios derivados de la situación económica del país, con un crecimiento moderado y la caída de la bolsa; y 3) seguir desarrollando las relaciones institucionales entre Euskadi y China (SPRI, 2015). La misión se reunió con media docena de empresas vascas ubicadas en China - Gamesa, Cie Automotive, Fagor Arrasate, Orkli, Batz y Ciakautxo-. Además, firmó un convenio con la Jiangsu Federation of Industry \& Commerce, organismo gubernamental sin ánimo de lucro formado por las entidades industriales y comerciales de la provincia de Jiangsu. «El objetivo es fortalecer los intercambios y cooperaciones en el ámbito comercial, empresarial e institucional, el establecimiento de contactos empresariales, la promoción de negocios e inversiones mutuas, el desarrollo de proyectos 
de cooperación, el establecimiento de contactos entre empresas y organizaciones, y la organización de ferias, seminarios, congresos, conferencias, misiones empresariales y sectoriales» (SPRI, 2015). Asimismo, visitó el CCPIT Shanghái (Council for the Promotion of International Trade Shanghai), una organización gubernamental para la promoción del comercio exterior, y la Cámara China de Comercio Internacional de Shanghái.

Para dar apoyo a las empresas vascas en su proceso de internacionalización, la CAV cuenta con un completo y complejo sistema público de apoyo que despliega servicios que cubren todas las fases de internacionalización (Gobierno Vasco, 2014a: 52). El Gobierno Vasco, las diputaciones forales y las cámaras de comercio suman a 100 profesionales para un presupuesto global de 30 millones de euros. Únicamente en el área de financiación la oferta del sistema vasco de internacionalización es más limitada, por lo que las empresas recurren a organismos estatales de internacionalización como el ICEX o la CESCE, o a herramientas generales de apoyo a la financiación industrial de Euskadi (Gobierno Vasco, 2014a: 52). En la actualidad, el sistema vasco de internacionalización empresarial tiene el doble reto de mejorar su apoyo a las empresas vascas, desarrollando una hoja de ruta común y actualizada del apoyo a la internacionalización, y de avanzar en la reordenación y optimización del propio sistema, adaptándolo a la realidad de los recursos públicos actuales y a la iniciativa privada en el ámbito de los servicios de internacionalización (Gobierno Vasco, 2014a: 52).

\subsection{Las características del Plan de Internacionalización}

El Plan de Internacionalización Empresarial 2014-2016, además de tener un alcance, unos objetivos y unos ejes estratégicos de actuación, se sustenta sobre una serie de principios que conviene mencionar (Gobierno Vasco, 2014a: 56):

- Acción y resultados a corto y medio plazo; es decir, por una parte, pretende contrarrestar los efectos de la crisis sobre las empresas vascas, y, por otra, aspira a reforzar el avance en la competitividad del tejido económico vasco en el medio plazo como vía esencial para la mejora permanente y sostenible.

- Apuesta por la industria productiva ya que es clave por su peso y su capacidad de arrastre, por ser el principal motor de generación de riqueza y empleo y la palanca principal de internacionalización y de $\mathrm{I}+\mathrm{D}+\mathrm{i}$ (Gobierno Vasco, 2014a: 56).

+ Perspectiva económica transversal, lo que significa que todos los ámbitos sectoriales concernidos por ese plan se conciben desde una visión empre- 
sarial de generación de riqueza, empleo y bienestar, sin olvidar las externalidades positivas que generan.

- Focalización de esfuerzos, lo que supone la necesidad de incrementar la eficiencia en el uso de los recursos públicos y la limitación de recursos que la situación y la propia dimensión de la CAV imponen. Esto lleva el Gobierno Vasco a focalizar sus políticas y acciones allá donde más impacto puedan generar y a evaluar constantemente dichos efectos (Gobierno Vasco, 2014a: 56).

- Impulso de cadenas complejas de actividad; es decir, el Gobierno Vasco quiere orientar sus políticas a fortalecer cadenas complejas de actividad económica, además de a fomentar las relaciones entre los distintos eslabones de dicha cadena o entre cadenas diferentes. Se trata de avanzar en una nueva fase de clusterización de la constelación de cadenas de valor interrelacionadas.

- Segmentación de políticas diseñando políticas y soluciones que se adapten a cada empresa o conjunto de empresas con características similares, considerando su estado de evolución y maduración, su tamaño y la actividad en que operan (Gobierno Vasco, 2014a: 56).

- Participación de las empresas, dado que los trabajadores son sus principales activos. El Plan desea apoyar procesos de refuerzo de la participación de los trabajadores en las organizaciones como mecanismo de mejora de la competitividad, generando proyectos compartidos a largo plazo.

+ Colaboración multiactor; es decir, cooperación interinstitucional y colaboración público-privada con vistas a lograr las metas propuestas, reinventando algunas de las fórmulas tradicionales que presentan ciertos síntomas de agotamiento (Gobierno Vasco, 2014a 56 ).

- Equilibrio territorial, pues este constituye una de las principales fortalezas de la CAV. El desarrollo urbano, rural y litoral de sus territorios, comarcas y municipios supone una prioridad para el Gobierno Vasco.

Sobre la base de estos principios cardinales, el Plan de Internacionalización Empresarial desea promover la inserción y competitividad internacional de las empresas vascas, y especialmente de las pymes, en el marco global, estableciendo un dispositivo de apoyo adaptado a sus capacidades y necesidades en el contexto de crisis actual, impulsando la apertura e internacionalización de la economía vasca, potenciando y consolidando el comercio exterior, contribuyendo al incremento de implantaciones vascas en el exterior y atrayendo inversión extranjera hacia Euskadi (Gobierno Vasco, 2014a:58). Además, dada su integración en la 
Estrategia Marco de Internacionalización 2020: Euskadi Basque Country, el Plan de Internacionalización Empresarial 2014-2016 desea promover una combinación de políticas de refuerzo inteligente de la internacionalización que actúen como palanca de competitividad y fortalecimiento del tejido empresarial vasco y de diversificación de sectores, mercados y riesgos (Gobierno Vasco, 2014a: 58).

En cuanto a los ejes estratégicos y las líneas de actuación de dicho Plan, apuesta por fijar condiciones y propiciar comportamientos que ayuden al tejido empresarial, siempre teniendo en cuenta la especificidad de cada empresa y sector e intentando fortalecer su posición competitiva en el ámbito internacional. Para alcanzar los objetivos fijados, el ejecutivo vasco ha definido una serie de estrategias de actuación prioritaria (Gobierno Vasco, 2014a: 58):

- La consolidación y el impulso del sistema de apoyo a la actividad exterior.

- El apoyo diferenciado para el acceso de las empresas vascas a mercados internacionales.

- El apoyo a sectores y clústeres para reforzar el posicionamiento internacional de los bienes y servicios vascos en el exterior.

- La generación y dinamización del capital humano para acometer los mercados internacionales.

- La capacitación y consolidación de inversiones extranjeras.

- La financiación y cooperación internacional y multilateral (Gobierno Vasco, 2014a: 58).

\section{Conclusión}

Aún es pronto para evaluar la eficacia del Plan de Internacionalización Empresarial 2014-2016 en particular y de la política de internacionalización del Gobierno Vasco en general. No en vano, dicha internacionalización se antoja ineludible en un contexto en el que el mercado europeo muestra signos de estancamiento como consecuencia de las políticas de austeridad implementadas por la mayoría de los Estados miembros de la Unión Europea, sinónimo de recortes del gasto público y de incremento de la presión fiscal, y de la política monetaria restrictiva llevada a cabo por el Banco Central Europeo, que se preocupa ante todo por luchar contra la inflación. La consecuencia de todo ello es que los mercados tradicionales de las empresas vascas, tales como el francés y, en menor medida, el alemán, han retrocedido, lo que ha limitado el potencial exportador de las empresas vascas hacia esos mercados.

En ese sentido, después de dos décadas de crecimiento económico prácticamente continuado que han permitido a la CAV alcanzar altos niveles de bienes- 
tar y acercarla a las regiones europeas con mayores niveles de riqueza, ese territorio se encuentra inmerso en una crisis que se prolonga desde hace varios años y que ha provocado la destrucción de más de 100.000 puestos de trabajo desde 2007 (Gobierno Vasco, 2014a: 18) y el agotamiento del tejido productivo. Las empresas vascas tienen dificultades para recuperar los niveles de actividad y de empleo anteriores a la crisis y para competir con garantías en los mercados globalizados (Gobierno Vasco, 2014a: 18). Euskadi parte de un retraso competitivo respecto a países y regiones de referencia europeos, lo que supone una restricción para la internacionalización de sus empresas. Por todo ello, las políticas de apoyo a la internacionalización empresarial del Gobierno Vasco deben ser coherentes con otras políticas públicas dirigidas a mejorar la competitividad de las empresas vascas (Gobierno Vasco, 2014a: 18).

No en vano, la política de internacionalización del Gobierno Vasco se enfrenta a restricciones legales y presupuestarias en virtud del nuevo marco de estabilidad presupuestaria (Gobierno Vasco, 2014a: 18). Las legislaciones europeas y estatales dirigidas a garantizar el equilibrio presupuestario y la sostenibilidad de las finanzas públicas, definidas principalmente por el Pacto de Estabilidad y de Crecimiento de la Unión Europea y el Pacto Fiscal del Estado, exigen cumplir con estrictos requerimientos de déficit y de deuda pública que limitan la capacidad de acción de las administraciones públicas (Gobierno Vasco, 2014a: 18). Las instituciones vascas, a pesar de gozar de mayor autonomía fiscal y financiera, están igualmente sujetas a requerimientos de estabilidad presupuestaria a través de acuerdos con el Estado, de modo que «su capacidad de acción al esfuerzo competitivo empresarial esté condicionada por la evolución de los ingresos fiscales, que crecerán moderadamente de acuerdo al crecimiento económico esperado» (Gobierno Vasco, 2014a: 18).

En cualquier caso, ya se beneficien o no de la ayuda prestada por el Gobierno Vasco vía el Plan de Internacionalización Empresarial 2014-2016, las empresas vascas están condenadas a internacionalizarse y China aparece como un mercado preferente por las oportunidades que ofrece, sin por ello infravalorar los obstáculos existentes (Aldama, 2014). 


\section{Bibliografía}

Aldama, Z. (2014): «El calvario chino», El País, 27 de enero de 2014.

Altuna, R. y Urteaga, E. (2014a): «Le modèle de gestion dans les coopératives de Mondragon: qualité, légitimation et changement institutionnel», Revue Internationale d'Economie Sociale, núm. 333, pp. 26-42.

Altuna, R. y Urteaga, E. (2014b): «La cooperativa Fagor Ederlan: el 'lean manufacturing' como modelo de gestión», CIRIEC: Revista de Economía Pública, Social y Cooperativa, núm. 82, pp. 29-64.

Álvarez, M. (2015): «Las empresas vascas pierden interés por implantarse en China», El Correo, 13 de abril de 2015.

Arriola, A. (2015): «Más servicios a la empresa vasca para su internacionalización», Revista APD: Asociación para el Progreso de la Dirección, núm. 305.

Basterra, J. (2008): «Más de cien empresas vascas producen bajo el epígrafe 'Made in China' desde los 90», Gara, 16 de noviembre de 2008.

Borgonjon, J. (2009): «China como mercado de consumo y servicios para empresas españolas», Boletín Económico de ICE, núm. 2972.

CEISS Consultores (2009): De Europa a Asia, un mundo lleno de posibilidades. Internacionalización de las empresas de la Comunidad Autónoma Vasca y Navarra. San Sebastián: CEISS.

Confebask (2014): «Principales rasgos económicos de 2014 y perspectivas 2015», Informe de Coyuntura Económica, diciembre de 2014.

Cuesta, M. (2015)ः «Guía rápida para entender la crisis china», $A B C, 2$ de septiembre de 2015.

Diez Mon, A. (2014): «Las empresas vascas triplican los procesos de internacionalización durante la crisis», Deia, 6 de octubre de 2014.

- (2015): «Los exportadores vascos superan con nota las turbulencias de China y Brasil», Noticias de Gipuzkoa, 28 de septiembre de 2015.

EFE (2011): «La SPRI abre una oficina en Pekín para apoyar las empresas vascas en China», $A B C, 26$ de abril de 2011.

- (2012): «Casi doscientas empresas vascas cuentan con presencia en China», El Mundo, 18 de septiembre de 2012.

EITB (2015): «Orbea y Fagor Industrial cierran sus plantas en China» < <ttp:// www.eitb.eus/es/noticias/economia/detalle/3137218/orbea-fagor-industrial-cierran-sus-plantas-china/>

Euskonews \& Media (2015): «Entrevista de Marian Elorza, directora de Internacionalización del Gobierno Vasco», Euskonews \& Media, núm. 348.

Gobierno Vasco (2014a): Plan de Internacionalización Empresarial 2014-2016. Vitoria: Gobierno Vasco. 
Gobierno Vasco (2014b): Estrategia Marco de Internacionalización 2014-2016: Euskadi Basque Country. Vitoria: Gobierno Vasco.

IBERChina (2016): «Las cinco mejores empresas españolas en China».<http:// www.iberchina.org/index.php/espahina-contenidos-38/894-las-cinco-mejores-empresas-espanolas-en-china>

LARrea, J.-L. (2008): «Internacionalización de la empresa vasca: retos desde la competitividad», Boletín de Estudios Económicos, vol. 63, núm. 193, pp. 59-82.

Lemoine, F. y Hernández, M. (2007): La economía china. Barcelona: Alianza.

Luzarraga, J.-M. (2006): La estrategia de empresas vascas en China: claves del éxito y retos existentes. Shanghái: Euskal Etxea Shanghai.

Oficina de Información Diplomática (2014): China. Madrid: Oficina de Información Diplomática del Ministerio de Asuntos Exteriores y de Cooperación.

Peters, S. (2010): «Riesgos y oportunidades de los mercados emergentes: los casos de India y China», Revista CIDOB d'afers internationals, núm. 89-90, pp. 127-148.

Santander Trade (2015): «China: política y economía», <https:// es.santandertrade.com/analizar-mercados/china/politica-y-economia $>$

SPRI (2009)ः «Presencia de empresas vascas en China y perspectivas». Shanghái* SPRI Shanghai Representative Office.

SPRI (2015): «El Grupo SPRI visita China para apoyar a las empresas vascas». $<$ http://www.spri.eus/es/actualidad-spri/noticias/internacionalizacion-elgrupo-spri-visita-china-para-apoyar-a-las-empresas-vascas\#sthash.mevariKu.dpuf>

Velasco, R. (1991): «Internacionalización de la empresa vasca: estrategias y políticas para los años 90», Ekonomiaz, núm. 20, pp. 228-243. 